\title{
ON THE BOUNDARY OF THE MODULI SPACES OF LOG HODGE STRUCTURES: TRIVIALITY OF THE TORSOR
}

\author{
TATSUKI HAYAMA
}

\begin{abstract}
This paper examines the moduli spaces of log Hodge structures introduced by Kato and Usui. This moduli space is a partial compactification of a discrete quotient of a period domain. This paper treats the following two cases: (A) where the period domain is Hermitian symmetric, and (B) where the Hodge structures are of the mirror quintic type. Especially it addresses a property of the torsor.
\end{abstract}

\section{$\S 1$. Introduction}

Let $\varphi:\left(\Delta^{*}\right)^{n} \rightarrow \Gamma \backslash D$ be a period map arising from a variation of Hodge structures with unipotent monodromies on the $n$-fold product of the punctured disk. Here $\Gamma$ is the image of $\pi_{1}\left(\left(\Delta^{*}\right)^{n}\right) \cong \mathbb{Z}^{n}$ by the monodromy representation; that is, $\Gamma$ is a free $\mathbb{Z}$-module. By Schmid's nilpotent orbit theorem $[\mathrm{S}]$, the behavior of the period map around the origin is approximated by a "nilpotent orbit." Then we add the set of nilpotent orbits to $\Gamma \backslash D$ as boundary points and extend the period map satisfying the following diagram:

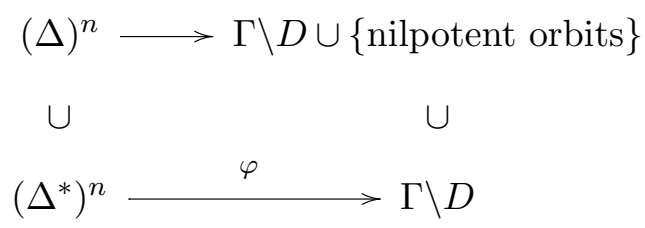

Here $\Gamma \backslash D$ is an analytic space and $\varphi$ is an analytic morphism. But, except in some cases, we have no way to endow the upper right one with an analytic structure.

Received February 24, 2009. Revised November 7, 2009. Accepted November 12, 2009. 2000 Mathematics Subject Classification. 32G20.

This work was supported by a Grant-in-Aid from the Japan Society for the Promotion of Science (JSPS).

(C) 2010 by The Editorial Board of the Nagoya Mathematical Journal 
In [KU] Kato and Usui endow it with a geometric structure as a "logarithmic manifold," and they treat the above diagram as a diagram in the category of logarithmic manifolds. Moreover, they define "polarized logarithmic Hodge" (PLH) structures, and they show that the upper right one is the moduli space of PLH. The result here is for the geometric structure of the moduli spaces of PLH in the following two cases:

(A) $D$ is a Hermitian symmetric space;

(B) polarized Hodge structures $(\mathrm{PH})$ are of weight $w=3$ and Hodge number $h^{p, q}=1(p+q=3, p, q \geq 0)$, and logarithms of the monodromy transformations are of type $N_{\alpha}$ in $[\mathrm{KU}, \S 12.3]$ (or type $\mathrm{II}_{1}$ in [GGK]).

Case (A) corresponds to degenerations of algebraic curves or K3 surfaces. This case is classical and well known. Case (B) corresponds to degenerations of certain Calabi-Yau threefolds, for instance, those occurring in the mirror quintic family. This case was studied recently. For example, Green, Griffiths, and Kerr ([GGK]) describe "Néron models" for VHS (variation of Hodge structure) of this type. Usui ([U]) shows a logarithmic Torelli theorem for this quintic mirror family.

\section{Construction of a moduli space of PLH}

To explain this result, this paper describes Kato and Usui's construction of the moduli space of PLH roughly (see Section 3 for details). Steps of the construction are given as follows.

Step 1. Define the nilpotent cone $\sigma$ and the set $D_{\sigma}$ of nilpotent orbits.

Step 2. Define the toric variety toric $\sigma$ and the space $E_{\sigma}$.

Step 3. Define the map $E_{\sigma} \rightarrow \Gamma \backslash D_{\sigma}$, and endow $\Gamma \backslash D_{\sigma}$ with a geometric structure by this map.

First, fix a point $s_{0} \in\left(\Delta^{*}\right)^{n}$, and let $\left(H_{s_{0}}, F_{s_{0}},\langle,\rangle_{s_{0}}\right)$ be the corresponding PH structure. The period domain $D$ is a homogeneous space for the real Lie group $G=\operatorname{Aut}\left(H_{s_{0}, \mathbb{R}},\langle,\rangle_{s_{0}}\right)$ and also an open $G$-orbit in the flag manifold $\check{D}$ (see Section 2 for details).

Step 1. Take the logarithms $N_{1}, \ldots, N_{n}$ of the monodromy transformations, and make the cone $\sigma$ in $\mathfrak{g}$ generated by them, which is called a nilpotent cone. By Schmid's nilpotent orbit theorem, there exists the limiting Hodge filtration $F_{\infty}$. We call the orbit $\exp \left(\sigma_{\mathbb{C}}\right) F_{\infty}$ in $\check{D}$ a nilpotent orbit. $D_{\sigma}$ is the set of all nilpotent orbits generated by $\sigma$.

Step 2. Take the monoid $\Gamma(\sigma):=\Gamma \cap \exp (\sigma)$. It determines the toric

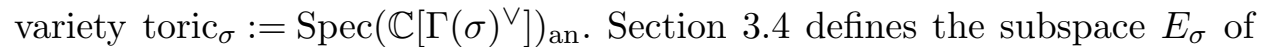
toric $_{\sigma} \times \check{D}$. 
Step 3. Section 3.5 defines the map $E_{\sigma} \rightarrow \Gamma \backslash D_{\sigma}$ (here $\left.\Gamma=\Gamma(\sigma)^{\mathrm{gp}}\right)$. By [KU, Theorem A], $E_{\sigma}$ and $\Gamma \backslash D_{\sigma}$ are logarithmic manifolds. Moreover, the map is a $\sigma_{\mathbb{C}}$-torsor in the category of logarithmic manifolds; that is, there exists a proper and free $\sigma_{\mathbb{C}^{-}}$-action on $E_{\sigma}$, and $E_{\sigma} \rightarrow \Gamma \backslash D_{\sigma}$ is isomorphic to $E_{\sigma} \rightarrow \sigma_{\mathbb{C}} \backslash E_{\sigma}$ in the category of logarithmic manifolds.

\section{Main result}

The main result is for properties of the torsor $E_{\sigma} \rightarrow \Gamma \backslash D_{\sigma}$.

- In case (A), the torsor is trivial (Theorem 5.6).

- In case (B), the torsor is nontrivial (Proposition 5.8).

In case (A), $\Gamma \backslash D_{\sigma}$ is just a toroidal partial compactification of $\Gamma \backslash D$ introduced by $[\mathrm{AMRT}]$. To show the triviality, Section 4 reviews the theory of bounded symmetric domains. Realization of $D$ as the Siegel domain of the third kind (4.2) is a key of the proof. This induces the triviality of $B(\sigma) \rightarrow \mathbb{B}(\sigma)$ (Lemma 5.1). By the triviality of this torsor, we show the triviality of $E_{\sigma} \rightarrow \Gamma \backslash D_{\sigma}$. We also describe a simple example (Example 5.7).

In case $(\mathrm{B}), D$ is not a Hermitian symmetric domain; that is, isotropy subgroups are not maximally compact. Fix a point $F_{0} \in D$, and take a maximally compact subgroup $K$ as in (5.2). Then $K F_{0}$ is a compact subvariety of $D$. Existence of such a variety (of positive dimension) is a distinction between the cases where the period domain is Hermitian symmetric and otherwise. The compact subvariety plays an important role in the proof.

\section{§2. Polarized Hodge structures and period domains}

Recall the definition of $\mathrm{PH}$ structures and of period domains. A Hodge structure of weight $w$ and of Hodge type $\left(h^{p, q}\right)$ is a pair $(H, F)$ consisting of a free $\mathbb{Z}$-module of rank $\sum_{p, q} h^{p, q}$ and of a decreasing filtration on $H_{\mathbb{C}}:=H \otimes \mathbb{C}$ satisfying the following conditions:

(H1) $\operatorname{dim}_{\mathbb{C}} F^{p}=\sum_{r \geq p} h^{r, w-r}$ for all $p$;

(H2) $H_{\mathbb{C}}=\bigoplus_{p+q} H^{p, q}\left(H^{p, q}:=F^{p} \cap \overline{F^{w-p}}\right)$.

A polarization $\langle$,$\rangle for a Hodge structure (H, F)$ of weight $w$ is a nondegenerate bilinear form on $H_{\mathbb{Q}}:=H \otimes \mathbb{Q}$, symmetric if $w$ is even and skewsymmetric if $w$ is odd, satisfying the following conditions:

(P1) $\left\langle F^{p}, F^{q}\right\rangle=0$ for $p+q>w$;

(P2) The Hermitian form

$$
H_{\mathbb{C}} \times H_{\mathbb{C}} \rightarrow \mathbb{C}, \quad(x, y) \mapsto\left\langle C_{F}(x), \bar{y}\right\rangle
$$

is positive definite. 
Here $\langle$,$\rangle is regarded as the natural extension to \mathbb{C}$-bilinear form, and $C_{F}$ is the Weil operator, which is defined by $C_{F}(x):=(\sqrt{-1})^{p-q} x$ for $x \in H^{p, q}$.

Fix a PH structure $\left(H_{0}, F_{0},\langle,\rangle_{0}\right)$ of weight $w$ and of Hodge type $\left(h^{p, q}\right)$. Define the set of all Hodge structures of this type

$$
D:=\left\{F \mid \begin{array}{l}
\left(H_{0}, F,\langle,\rangle_{0}\right) \text { is } \mathrm{PH} \\
\text { of weight } w \text { and of Hodge type }\left(h^{p, q}\right)
\end{array}\right\} .
$$

$D$ is called a period domain. Moreover, we have the flag manifold

$$
\check{D}:=\left\{F \mid \begin{array}{r}
\left(H_{0}, F,\langle,\rangle_{0}\right) \\
\text { satisfy the conditions } \\
(\mathrm{H} 1),(\mathrm{H} 2), \text { and }(\mathrm{P} 1)
\end{array}\right\} .
$$

$\check{D}$ is called the compact dual of $D$. D is contained in $\check{D}$ as an open subset. $D$ and $\check{D}$ are homogeneous spaces under the natural actions of $G$ and $G_{\mathbb{C}}$, respectively, where $G:=\operatorname{Aut}\left(H_{0, \mathbb{R}},\langle,\rangle_{0}\right)$ and $G_{\mathbb{C}}$ is the complexification of $G$. $G$ is a classical group such that

$$
G \cong \begin{cases}S p(h, \mathbb{R}) /\{ \pm 1\} & \text { if } w \text { is odd } \\ S O\left(h_{\text {odd }}, h_{\text {even }}\right) & \text { if } w \text { is even }\end{cases}
$$

where $2 h=\operatorname{rank} H_{0}, h_{\text {odd }}=\sum_{\text {p:odd }} h^{p, q}$, and $h_{\text {even }}=\sum_{\text {p:even }} h^{p, q}$. The isotropy subgroup of $G$ at $F_{0}$ is isomorphic to

$$
\begin{cases}\prod_{p \leq m} U\left(h^{p, q}\right) & \text { if } w=2 m+1, \\ \prod_{p<m} U\left(h^{p, q}\right) \times S O\left(h^{m, m}\right) & \text { if } w=2 m .\end{cases}
$$

They are compact subgroups of $G$ but not maximal compact in general; $D$ is a Hermitian symmetric domain if and only if the isotropy subgroup is a maximally compact subgroup, that is, one of the following is satisfied:

(1) $w=2 m+1, h^{p, q}=0$ unless $p=m+1, m$;

(2) $w=2 m, h^{p, q}=1$ for $p=m+1, m-1, h^{m, m}$ is arbitrary, $h^{p, q}=0$ otherwise;

(3) $w=2 m, h^{p, q}=1$ for $p=m+a, m+a-1, m-a, m-a+1$ for some $a \geq 2, h^{p, q}=0$ otherwise.

In case (1), $D$ is a Hermitian symmetric domain of type III. In case (2) or (3), an irreducible component of $D$ is a Hermitian symmetric domain of type IV. 


\section{$\S 3$. Moduli spaces of polarized log Hodge structures}

This section reviews some basic facts in [KU]. First, it introduces $D_{\Sigma}$, a set of nilpotent orbits associated with a fan $\Sigma$ consisting of nilpotent cones. Second, for some subgroup $\Gamma$ in $G_{\mathbb{Z}}$, we endow $\Gamma \backslash D_{\Sigma}$ with a geometric structure. Finally, we see some fundamental properties of $\Gamma \backslash D_{\Sigma}$, which are among the main results of $[\mathrm{KU}]$.

\subsection{Nilpotent orbits}

A nilpotent cone $\sigma$ is a strongly convex and finitely generated rational polyhedral cone in $\mathfrak{g}:=\operatorname{Lie} G$ whose generators are nilpotent and commute with each other. For $A=\mathbb{R}, \mathbb{C}$, denote by $\sigma_{A}$ the $A$-linear span of $\sigma$ in $\mathfrak{g}_{A}$.

Definition 3.1. Let $\sigma=\sum_{j=1}^{n} \mathbb{R}_{\geq 0} N_{j}$ be a nilpotent cone, and let $F \in \check{D}$. $\operatorname{Exp}\left(\sigma_{\mathbb{C}}\right) F \subset \check{D}$ is a $\sigma$-nilpotent orbit if it satisfies the following conditions:

(1) $\operatorname{Exp}\left(\sum_{j} i y_{j} N_{j}\right) F \in D$ for all $y_{j} \gg 0$;

(2) $N F^{p} \subset F^{p-1}$ for all $p \in \mathbb{Z}$ and for all $N \in \sigma$.

Condition (2) says the map $\mathbb{C}^{n} \rightarrow \check{D}$ given by $\left(z_{j}\right) \mapsto \sum_{j} \exp \left(z_{j} N_{j}\right) F$ is horizontal. Let $\Sigma$ be a fan consisting of nilpotent cones. Define the set of nilpotent orbits

$$
D_{\Sigma}:=\{(\sigma, Z) \mid \sigma \in \Sigma, Z \text { is a } \sigma \text {-nilpotent orbit }\}
$$

For a nilpotent cone $\sigma$, the set of faces of $\sigma$ is a fan, and we abbreviate $D_{\{\text {faces of } \sigma\}}$ as $D_{\sigma}$.

\subsection{Subgroups in $G_{\mathbb{Z}}$ compatible with a fan}

Let $\Gamma$ be a subgroup of $G_{\mathbb{Z}}$, and let $\Sigma$ be a fan of nilpotent cones. Say $\Gamma$ is compatible with $\Sigma$ if

$$
\operatorname{Ad}(\gamma)(\sigma) \in \Sigma,
$$

for all $\gamma \in \Gamma$ and for all $\sigma \in \Sigma$. Then $\Gamma$ acts on $D_{\Sigma}$ if $\Gamma$ is compatible with $\Sigma$. Moreover, we say $\Gamma$ is strongly compatible with $\Sigma$ if it is compatible with $\Sigma$ and for all $\sigma \in \Sigma$ there exists $\gamma_{1}, \ldots, \gamma_{n} \in \Gamma(\sigma):=\Gamma \cap \exp (\sigma)$ such that

$$
\sigma=\sum_{j} \mathbb{R}_{\geq 0} \log \left(\gamma_{j}\right)
$$


3.3. Varieties toric ${ }_{\sigma}$ and torus $_{\sigma}$

Let $\Sigma$ be a fan, and let $\Gamma$ be a subgroup of $G_{\mathbb{Z}}$ which is strongly compatible with $\Sigma$. We have toric varieties associated with the monoid $\Gamma(\sigma)$ such that

$$
\begin{aligned}
& \operatorname{toric}_{\sigma}:=\operatorname{Spec}\left(\mathbb{C}\left[\Gamma(\sigma)^{\vee}\right]\right)_{\mathrm{an}} \cong \operatorname{Hom}\left(\Gamma(\sigma)^{\vee}, \mathbb{C}\right), \\
& \text { torus }_{\sigma}:=\operatorname{Spec}\left(\mathbb{C}\left[\Gamma(\sigma)^{\vee g p}\right]\right)_{\mathrm{an}} \cong \operatorname{Hom}\left(\Gamma(\sigma)^{\vee \mathrm{gp}}, \mathbb{G}_{m}\right) \cong \mathbb{G}_{m} \otimes \Gamma(\sigma)^{\mathrm{gp}},
\end{aligned}
$$

where $\mathbb{C}$ is regarded as a semigroup via multiplication and the above homomorphisms are of semigroups. As in $[\mathrm{F}, \S 2.1]$, we choose the distinguished point

$$
x_{\tau}: \Gamma(\sigma)^{\vee} \rightarrow \mathbb{C} ; \quad u \mapsto \begin{cases}1 & \text { if } u \in \Gamma(\tau)^{\perp}, \\ 0 & \text { otherwise, }\end{cases}
$$

for a face $\tau$ of $\sigma$. Then toric $\sigma$ can be decomposed as

$$
\operatorname{toric}_{\sigma}=\bigsqcup_{\tau \prec \sigma}\left(\text { torus }_{\sigma} \cdot x_{\tau}\right) \text {. }
$$

For $q \in$ toric $_{\sigma}$, there exists $\sigma(q) \prec \sigma$ such that $q \in$ torus $_{\sigma} \cdot x_{\sigma(q)}$. By the surjective homomorphism

$$
\mathbf{e}: \sigma_{\mathbb{C}} \rightarrow \operatorname{torus}_{\sigma} \cong \mathbb{G}_{m} \otimes \Gamma(\sigma)^{\mathrm{gp}} ; \quad w \log (\gamma) \mapsto \exp (2 \pi \sqrt{-1} w) \otimes \gamma
$$

$q$ can be written as

$$
q=\mathbf{e}(z) \cdot x_{\sigma(q)}
$$

Here $\operatorname{ker}(\mathbf{e})=\log \left(\Gamma(\sigma)^{\mathrm{gp}}\right)$, and $z$ is determined uniquely modulo $\log \left(\Gamma(\sigma)^{\mathrm{gp}}\right)+$ $\sigma(q) \mathbb{C}$.

\subsection{Spaces $\check{E}_{\sigma}$ and $E_{\sigma}$}

Define the analytic space $\check{E}_{\sigma}:=$ toric $_{\sigma} \times \check{D}$, and endow $\check{E}_{\sigma}$ with the logarithmic structure $M_{E_{\sigma}}$ by the inverse image of canonical logarithmic structure on toric $\sigma$ (see $[\mathrm{K}])$. Define the subspace of $\check{E}_{\sigma}$ as

$$
E_{\sigma}:=\left\{(q, F) \in \check{E}_{\sigma} \mid \exp \left(\sigma(q)_{\mathbb{C}}\right) \exp (z) F \text { is } \sigma(q) \text {-nilpotent orbit }\right\},
$$

where $z$ is an element such that $q=\mathbf{e}(z) \cdot x_{\sigma(q)}$. The set $E_{\sigma}$ is well defined. The topology of $E_{\sigma}$ is the "strong topology" in $\check{E}_{\sigma}$, which is defined in [KU, $\S 3.1]$, and $\mathcal{O}_{E_{\sigma}}\left(\right.$ resp., $\left.M_{E_{\sigma}}\right)$ is the inverse image of $\mathcal{O}_{\check{E}_{\sigma}}\left(\right.$ resp., $M_{\check{E}_{\sigma}}$ ). Then 
$E_{\sigma}$ is a logarithmic local ringed space. Note that $E_{\sigma}$ is not an analytic space in general.

\subsection{The structure of $\Gamma \backslash D_{\Sigma}$}

We define the canonical map

$$
\begin{aligned}
& E_{\sigma} \rightarrow \Gamma(\sigma)^{\mathrm{gp}} \backslash D_{\sigma}, \\
& (q, F) \mapsto\left(\sigma(q), \exp \left(\sigma(q)_{\mathbb{C}}\right) \exp (z) F\right) \bmod \Gamma(\sigma)^{\mathrm{gp}},
\end{aligned}
$$

where $q=\mathbf{e}(z) \cdot x_{\sigma(q)}$ as in (3.2). This map is well defined. Endow $\Gamma \backslash D_{\Sigma}$ with the strongest topology for which the composite maps $\pi_{\sigma}: E_{\sigma} \rightarrow \Gamma(\sigma)^{\mathrm{gp}} \backslash$ $D_{\sigma} \rightarrow \Gamma \backslash D_{\Sigma}$ are continuous for all $\sigma \in \Sigma$. Endow $\Gamma \backslash D_{\Sigma}$ with $\mathcal{O}_{\Gamma \backslash D_{\Sigma}}$ (resp., $\left.M_{\Gamma \backslash D_{\Sigma}}\right)$ as follows:

$$
\begin{gathered}
\left.\mathcal{O}_{\Gamma \backslash D_{\Sigma}}(U) \text { (resp., } M_{\Gamma \backslash D_{\Sigma}}(U)\right) \\
:=\left\{\operatorname{map} f: U \rightarrow \mathbb{C} \mid f \circ \pi_{\sigma} \in \mathcal{O}_{E_{\sigma}}\left(\pi_{\sigma}^{-1}(U)\right)\right. \\
\left.\quad\left(\operatorname{resp} ., M_{E_{\sigma}}\left(\pi_{\sigma}^{-1}(U)\right)\right)(\forall \sigma \in \Sigma)\right\}
\end{gathered}
$$

for any open set $U$ of $\Gamma \backslash D_{\Sigma}$. As for $E_{\sigma}$, note that $\Gamma \backslash D_{\Sigma}$ is a logarithmic local ringed space but is not an analytic space in general. Kato and Usui introduce "logarithmic manifolds" as generalized analytic spaces (see [KU, $\S 3.5]$ ), and they show the following geometric properties of $\Gamma \backslash D_{\Sigma}$.

TheOREM 3.2. ([KU, Theorem A]) Let $\Sigma$ be a fan of nilpotent cones, and let $\Gamma$ be a subgroup of $G_{\mathbb{Z}}$ which is strongly compatible with $\Sigma$. Then we have the following.

(1) $E_{\sigma}$ is a logarithmic manifold.

(2) If $\Gamma$ is neat (i.e., the subgroup of $\mathbb{G}_{m}$ generated by all the eigenvalues of all $\gamma \in \Gamma$ is torsion free), $\Gamma \backslash D_{\Sigma}$ is also a logarithmic manifold.

(3) Let $\sigma \in \Sigma$, and define the action of $\sigma_{\mathbb{C}}$ on $E_{\sigma}$ over $\Gamma(\sigma)^{\mathrm{gp}} \backslash D_{\sigma}$ by

$$
a \cdot(q, F):=(\mathbf{e}(a) q, \exp (-a) F) \quad\left(a \in \sigma_{\mathbb{C}},(q, F) \in E_{\sigma}\right) .
$$

Then $E_{\sigma} \rightarrow \Gamma(\sigma)^{\mathrm{gp}} \backslash D_{\sigma}$ is a $\sigma_{\mathbb{C}}$-torsor in the category of logarithmic manifold.

(4) $\Gamma(\sigma)^{\mathrm{gp}} \backslash D_{\sigma} \rightarrow \Gamma \backslash D_{\Sigma}$ is open and locally an isomorphism of logarithmic manifold. 
In [KU, §2.4], Kato and Usui introduce "polarized log Hodge structures" and show that $\Gamma \backslash D_{\Sigma}$ is a fine moduli space of PLH structures if $\Gamma$ is neat ([KU, Theorem B] $)$.

\section{$\S 4$. The structure of bounded symmetric domains}

In this section we recall some basic facts on Hermitian symmetric domains (for more detail, see [AMRT, Chapter III], [N, appendix]). We define Satake boundary components and show that a Hermitian symmetric domain is a family of tube domains parametrized by a vector bundle over a Satake boundary component. This domain is called a Siegel domain of the third kind.

\subsection{Satake boundary components}

Let $D$ be a Hermitian symmetric domain. Then $\operatorname{Aut}(D)$ is a real Lie group, and the identity component $G$ of $\operatorname{Aut}(D)$ acts on $D$ transitively. Fix a base point $o \in D$. The isotropy subgroup $K$ at $o$ is a maximally compact subgroup of $G$. Let $s_{o}$ be a symmetry at $o$, and let

$$
\begin{aligned}
\mathfrak{g} & :=\operatorname{Lie}(G), \quad \mathfrak{k}:=\operatorname{Lie}(K), \\
\mathfrak{p} & :=\text { the subspace of } \mathfrak{g} \text { where } s_{O}=-\mathrm{Id} .
\end{aligned}
$$

Then we have a Cartan decomposition

$$
\mathfrak{g}=\mathfrak{k} \oplus \mathfrak{p} .
$$

$\mathfrak{p}$ is isomorphic to the tangent space to $D$ at $o$. Let $J$ be a complex structure on $\mathfrak{p}$, and let

$$
\begin{aligned}
& \mathfrak{p}_{+}:=\sqrt{-1} \text {-eigenspace for } J \text { in } \mathfrak{p}_{\mathbb{C}} \\
& \mathfrak{p}_{-}:=-\sqrt{-1} \text {-eigenspace for } J \text { in } \mathfrak{p}_{\mathbb{C}}
\end{aligned}
$$

Here $\mathfrak{p}_{+}$and $\mathfrak{p}_{-}$are abelian subalgebras of $\mathfrak{g}_{\mathbb{C}}$. Then we have the HarishChandra embedding map $D \rightarrow \mathfrak{p}_{+}$whose image is a bounded domain.

Definition 4.1. A Satake boundary component of $D$ is an equivalence class in $\bar{D}$, the topological closure of $D$ in $\mathfrak{p}_{+}$, under the equivalence relation generated by $x \sim y$ if there exists a holomorphic map

$$
\lambda:\{z \in \mathbb{C}|| z \mid<1\} \longrightarrow \mathfrak{p}_{+}
$$

such that $\operatorname{Im}(\lambda) \subset \bar{D}$ and $x, y \in \operatorname{Im}(\lambda)$. 
It is known that Satake boundary components are also Hermitian symmetric domains. Let $S$ be a Satake boundary component. Define

$$
\begin{aligned}
N(S) & :=\{g \in G \mid g S=S\} \\
W(S) & :=\text { unipotent radical of } N(S), \\
U(S) & :=\text { center of } W(S)
\end{aligned}
$$

These groups have the following properties.

Proposition 4.2. (1) $N(S)$ acts on $D$ transitively.

(2) There exists an abelian Lie subalgebra $\underline{v}(S) \subset \mathfrak{g}$ such that Lie $W(S)=$ $\underline{v}(S)+\operatorname{Lie} U(S)$.

(3) $W(S) / U(S)$ is an abelian Lie group which is isomorphic to $V(S):=$ $\exp \underline{v}(S)$.

Here $S$ is called rational if $N(S)$ is defined over $\mathbb{Q}$. If $S$ is rational, then $V(S)$ and $U(S)$ are also defined over $\mathbb{Q}$.

\subsection{Siegel domain of third kind}

Define a subspace of $\check{D}$

$$
D(S):=U(S)_{\mathbb{C}} \cdot D=\bigcup_{g \in U(S)_{\mathbb{C}}} g \cdot D,
$$

where $U(S)_{\mathbb{C}}:=U(S) \otimes \mathbb{C}$. By Proposition 4.2(1) and the fact that $U(S)$ is a normal subgroup, $N(S) U(S)_{\mathbb{C}}$ acts on $D(S)$ transitively. Choose the base point $o_{S}$ as in [AMRT, §4.2]. The isotropy subgroup $I$ of $G$ at $o_{S}$ is contained in $N(S)$. Then we have a map

$$
\Psi_{S}: D(S) \cong N(S) U(S)_{\mathbb{C}} / I \rightarrow N(S) U(S)_{\mathbb{C}} / N(S) \cong U(S),
$$

where the last isomorphism takes imaginary part. By [AMRT, §4.2, Theorem 1], we have an open homogeneous self-adjoint cone $C(S) \subset U(S)$ such that $\Psi_{S}^{-1}(C(S))=D$.

Theorem 4.3. (1) $U(S)_{\mathbb{C}}$ acts freely on $D(S) . D(S) \rightarrow U(S)_{\mathbb{C}} \backslash D(S)$ is a trivial principal homogeneous bundle.

(2) $V(S)$ acts freely on $U(S)_{\mathbb{C}} \backslash D(S) \cdot V(S) \backslash\left(U(S)_{\mathbb{C}} \backslash D(S)\right) \cong S$, and the quotient map $D(S) \rightarrow S$ is a complex vector bundle (although $V(S)$ is real). Moreover, it is trivial. 
(3) By (1) and (2), we have a trivialization

$$
D(S) \cong S \times \mathbb{C}^{k} \times U(S)_{\mathbb{C}}
$$

In this product representation, we have

$$
\Psi_{S}(x, y, z)=\operatorname{Im} z-h_{x}(y, y)
$$

where $h_{x}$ is a real-bilinear quadratic form $\mathbb{C}^{k} \times \mathbb{C}^{k} \rightarrow U(S)$ depending realanalytically on $x$.

Thus we have

$$
D \cong\left\{(x, y, z) \in S \times \mathbb{C}^{(g-k) k} \times U(S)_{\mathbb{C}} \mid \operatorname{Im} z \in C(S)+h_{x}(y, y)\right\} .
$$

\section{$\S 5$. Main result}

\subsection{The case where $E_{\sigma} \rightarrow \Gamma(\sigma)^{\mathrm{gp}} \backslash D_{\sigma}$ is trivial}

In this section, assume that $D$ is a period domain and also a Hermitian symmetric domain. The purpose is to show Theorem 5.6. The main theorem for the case where $D$ is the upper half-plane is described in Example 5.7.

Let $S$ be a Satake rational boundary component of $D$, and let $\sigma$ be a nilpotent cone included in $\operatorname{Lie}(U(s))$. First, let us show the triviality of the torsor for such a cone. Set

$$
B(\sigma):=\exp \left(\sigma_{\mathbb{C}}\right) \cdot D \subset \check{D}, \quad \mathbb{B}(\sigma):=\exp \left(\sigma_{\mathbb{C}}\right) \backslash B(\sigma) .
$$

Here $B(\sigma), \mathbb{B}(\sigma)$ are defined by Carlson, Cattani, and Kaplan ([CCK]) from the point of view of mixed Hodge theory.

LEMMA 5.1. $B(\sigma) \rightarrow \mathbb{B}(\sigma)$ is a trivial principal bundle with fiber $\exp \left(\sigma_{\mathbb{C}}\right)$.

Proof. Here $\exp \left(\sigma_{\mathbb{C}}\right)$ is a sub-Lie group of $U(S)_{\mathbb{C}}$, and $U(S)_{\mathbb{C}}$ is abelian. By Theorem 4.3(1), $D(S) \rightarrow \exp \left(\sigma_{\mathbb{C}}\right) \backslash D(S)$ is a trivial principal bundle. Furthermore, the following diagram is commutative:

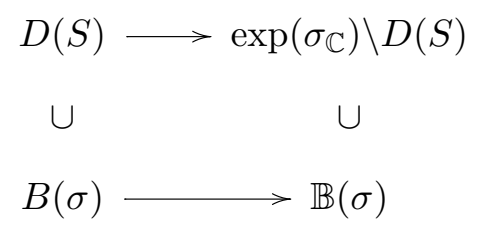

Then $B(\sigma) \rightarrow \mathbb{B}(\sigma)$ is trivial. 
Now let us describe a trivialization of $B(\sigma)$ over $\mathbb{B}(\sigma)$ explicitly. Take a complementary sub-Lie group $Z_{\sigma}$ of $\exp \left(\sigma_{\mathbb{C}}\right)$ in $U(S)_{\mathbb{C}}$. By $(4.1)$, we have a decomposition of $D(S)$ as $D(S) \cong S \times \mathbb{C}^{k} \times Z_{\sigma} \times \exp \left(\sigma_{\mathbb{C}}\right)$. Here

$$
\exp \left(\sigma_{\mathbb{C}}\right) \backslash D(S) \cong S \times \mathbb{C}^{k} \times Z_{\sigma}
$$

and the decomposition of $D(S)$ makes a trivialization of $D(S)$ over $\exp \left(\sigma_{\mathbb{C}}\right) \backslash$ $D(S)$. Via (5.1), we have $Y \subset S \times \mathbb{C}^{k} \times Z_{\sigma}$ satisfying the following commutative diagram:

$$
\begin{array}{ccc}
\exp \left(\sigma_{\mathbb{C}}\right) \backslash D(S) & \cong & S \times \mathbb{C}^{k} \times Z_{\sigma} \\
\cup & & \cup \\
\mathbb{B}(\sigma) & \cong & Y
\end{array}
$$

In fact, $Y$ is the image of $D$ via the projection $D(S) \rightarrow S \times \mathbb{C}^{k} \times Z_{\sigma}$. Then $B(\sigma) \cong \exp \left(\sigma_{\mathbb{C}}\right) \times Y$ is a trivialization of $B(\sigma)$ over $\mathbb{B}(\sigma)$.

Let $\Gamma$ be a subgroup of $G_{\mathbb{Z}}$ which is strongly compatible with $\sigma$. Let us think about a quotient trivial bundle $\Gamma(\sigma)^{\mathrm{gp}} \backslash B(\sigma) \rightarrow \mathbb{B}(\sigma)$. Its fiber is the quotient of $\exp \left(\sigma_{\mathbb{C}}\right)$ by the lattice $\Gamma(\sigma)^{\mathrm{gp}}$. Since $\exp \left(\sigma_{\mathbb{C}}\right)$ is a unipotent and abelian Lie group, $\sigma_{\mathbb{C}} \cong \exp \left(\sigma_{\mathbb{C}}\right)$. Via this isomorphism, the lattice action on $\exp \left(\sigma_{\mathbb{C}}\right)$ is equivalent to the lattice action on $\sigma_{\mathbb{C}}$ by $\log \left(\Gamma(\sigma)^{\mathrm{gp}}\right)$. Then the fiber is isomorphic to

$$
\sigma_{\mathbb{C}} / \log \left(\Gamma(\sigma)^{\mathrm{gp}}\right)=\sigma_{\mathbb{C}} / \operatorname{ker}(\mathbf{e}) \cong \operatorname{torus}_{\sigma}
$$

by (3.1). By the canonical torus action, $\Gamma(\sigma)^{\mathrm{gp}} \backslash B(\sigma) \rightarrow \mathbb{B}(\sigma)$ is also a principal torus ${ }_{\sigma}$-bundle whose trivialization is given by

$$
\operatorname{torus}_{\sigma} \times Y \stackrel{\sim}{\rightarrow} \Gamma(\sigma)^{\mathrm{gp}} \backslash B(\sigma) ; \quad(\mathbf{e}(z), F) \mapsto \exp (z) F \quad \bmod \Gamma(\sigma)^{\mathrm{gp}},
$$

where we regard $Y$ as a subset of $\check{D}$ via (4.1). By the definition of $E_{\sigma}$, we have the following.

LEMma 5.2. The following diagram is commutative:

$$
\begin{array}{ccc}
\Gamma(\sigma)^{\mathrm{gp} \backslash B(\sigma)} & \cong \quad \operatorname{torus}_{\sigma} \times Y \\
\cup & \cup \\
\Gamma(\sigma)^{\mathrm{gp} \backslash D} & \left.\cong \text { torus }_{\sigma} \times Y\right) \cap E_{\sigma}
\end{array}
$$


By the torus embedding torus ${ }_{\sigma} \hookrightarrow$ toric $_{\sigma}$, we can construct the associated bundle

$$
\left(\Gamma(\sigma)^{\mathrm{gp}} \backslash B(\sigma)\right)_{\sigma}:=\left(\Gamma(\sigma)^{\mathrm{gp}} \backslash B(\sigma)\right) \times_{\text {torus }_{\sigma}} \text { toric }_{\sigma}
$$

and define

$$
\left(\Gamma(\sigma)^{\mathrm{gp}} \backslash D\right)_{\sigma}:=\text { interior of the closure of } \Gamma(\sigma)^{\mathrm{gp}} \backslash D \text { in }\left(\Gamma(\sigma)^{\mathrm{gp}} \backslash B(\sigma)\right)_{\sigma} \text {. }
$$

This is a toroidal partial compactification associated with $\sigma$.

Lemma 5.3. The following diagram is commutative:

$$
\begin{aligned}
& \left(\Gamma(\sigma)^{\mathrm{gp}} \backslash B(\sigma)\right)_{\sigma} \cong \operatorname{toric}_{\sigma} \times Y \\
& \left(\Gamma(\sigma)^{\mathrm{gp}} \backslash D\right)_{\sigma} \cong\left(\text { toric }_{\sigma} \times Y\right) \cap E_{\sigma}
\end{aligned}
$$

Proof. For $(q, F) \in$ toric $_{\sigma} \times Y,(q, F) \in E_{\sigma}$ if and only if $\exp \left(\sigma(q)_{\mathbb{C}}\right) \times$ $\exp (z) F$ is a $\sigma(q)$-nilpotent orbit, where $q=\exp (z) x_{\sigma(q)}$ as in (3.2). Since $D$ is Hermitian symmetric, the horizontal tangent bundle of $D$ coincides with the tangent bundle of $D$. Then the condition of Definition 3.1(2) is trivially satisfied. Let $\left\{N_{i}\right\}$ be a set of rational nilpotent elements generating $\sigma(q)$. Then $(q, F) \in E_{\sigma}$ if and only if $\exp \left(\sum_{j} y_{j} N_{j}\right) \exp (z) F \in D$; that is,

$$
\left(\mathbf{e}\left(\sum_{j} y_{j} N_{j}+z\right), F\right) \in\left(\operatorname{torus}_{\sigma} \times Y\right) \cap E_{\sigma}
$$

for all $y_{j}$ such that $\operatorname{Im}\left(y_{j}\right) \gg 0$. In toric $\sigma$,

$$
\lim _{\operatorname{Im}\left(y_{j}\right) \rightarrow \infty} \mathbf{e}\left(\sum_{j} y_{j} N_{j}+z\right)=\mathbf{e}(z) x_{\sigma(q)}=q .
$$

Then $(q, F)$ is in the interior of the closure of $\left(\operatorname{torus}_{\sigma} \times Y\right) \cap E_{\sigma}$.

The map ( toric $\left._{\sigma} \times Y\right) \cap E_{\sigma} \hookrightarrow E_{\sigma} \rightarrow \Gamma(\sigma)^{\mathrm{gp}} \backslash D_{\sigma}$ is bijective. By [KU, Remark 8.2.7], $E_{\sigma}$ is an open set in $\check{E}_{\sigma}$. Then $E_{\sigma} \rightarrow \Gamma(\sigma)^{\mathrm{gp}} \backslash D_{\sigma}$ is a $\sigma_{\mathbb{C}^{-}}$ torsor in the category of analytic spaces, and

$$
\text { (toric } \left._{\sigma} \times Y\right) \cap E_{\sigma} \cong \sigma_{\mathbb{C}} \backslash E_{\sigma} \cong \Gamma(\sigma)^{\mathrm{gp}} \backslash D_{\sigma} .
$$


Thus (toric to $\left._{\sigma} \times Y\right) \cap E_{\sigma} \hookrightarrow E_{\sigma}$ gives a section of the torsor $E_{\sigma} \rightarrow \Gamma(\sigma)^{\mathrm{gp}} \backslash D_{\sigma}$; that is, $E_{\sigma} \rightarrow \Gamma(\sigma)^{\mathrm{gp}} \backslash D_{\sigma}$ is trivial.

Next let us show that $E_{\sigma} \rightarrow \Gamma(\sigma)^{\mathrm{gp}} \backslash D_{\sigma}$ is trivial for all nilpotent cones $\sigma$. Let $\Gamma=G_{\mathbb{Z}}$. By [AMRT, Chapter II], there exists a $\Gamma$-admissible collection of fans $\Sigma=\{\Sigma(S)\}_{S}$ where $\Sigma(S)$ is a fan in $\overline{C(S)}$ for every Satake rational boundary component $S$. Taking the logarithm, we identify $\Sigma$ with the collection of fans in $\mathfrak{g}$ which are strongly compatible with $\Gamma$. We show that $\Sigma$ is large enough to cover all nilpotent cones; that is, $\Sigma$ is a complete fan.

Let $U(S)_{\mathbb{Z}}=U(S) \cap \Gamma$. To obtain $U(S)_{\mathbb{Z}} \backslash D_{\sigma}$, we should confirm the following proposition.

\section{Proposition 5.4. Generators of $Z_{\sigma}$ can be taken in $G_{\mathbb{Z}}$.}

Proof. $\Gamma(\sigma)^{\mathrm{gp}}$ is saturated in $U(S)_{\mathbb{Z}}$; that is, if $g \in U(S)_{\mathbb{Z}}$ and $g^{n} \in \Gamma(\sigma)^{\mathrm{gp}}$ for some $n \geq 1$, then $g \in \Gamma(\sigma)^{\mathrm{gp}}$. Then $U(S)_{\mathbb{Z}} / \Gamma(\sigma)^{\mathrm{gp}}$ is a free module, and there exists a subgroup $Z_{\sigma, \mathbb{Z}}$ in $U(S)_{\mathbb{Z}}$ such that $U(S)_{\mathbb{Z}}=\Gamma(\sigma)^{\mathrm{gp}} \oplus Z_{\sigma, \mathbb{Z}}$. Hence we have $U(S)_{\mathbb{C}}=\exp \sigma_{\mathbb{C}} \oplus\left(Z_{\sigma, \mathbb{Z}} \otimes \mathbb{C}\right)$.

Gluing $U(S)_{\mathbb{Z}} \backslash D_{\sigma}=Z_{\sigma, \mathbb{Z}} \backslash\left(\Gamma(\sigma)^{\mathrm{gp}} \backslash D_{\sigma}\right)$ for $\sigma \in \Sigma(S)$, we have $U(S)_{\mathbb{Z}} \backslash$ $D_{\Sigma(S)}$ as a toroidal partial compactification in the direction $S$. Then we obtain a compact variety $\Gamma \backslash D_{\Sigma}$ by $\left[\right.$ AMRT]. (Take the quotient by $N(S)_{\mathbb{Z}} /$ $U(S)_{\mathbb{Z}}$, and glue neighborhoods of boundaries of $\left(N(S)_{\mathbb{Z}} / U(S)_{\mathbb{Z}}\right) \backslash\left(U(S)_{\mathbb{Z}} \backslash\right.$ $\left.D_{\Sigma(S)}\right)$ with $\left.\Gamma \backslash D.\right)$

On the other hand, we have the following proposition.

Proposition 5.5. ([KU, Proposition 12.6.4]) Let $\Gamma$ be a subgroup of $G_{\mathbb{Z}}$, and let $\Sigma$ be a fan which is strongly compatible with $\Gamma$. Assume that $\Gamma \backslash D_{\Sigma}$ is compact. Then $\Sigma$ is complete.

The precise definition of complete fan is given in [KU]. An important property of a complete fan $\Sigma$ is the following: if there exists $Z \subset \check{D}$ such that $(\sigma, Z)$ is a nilpotent orbit, then $\sigma \in \Sigma$. Now the $\Gamma$-admissible collection of fans $\Sigma$ is complete since $\Gamma \backslash D_{\Sigma}$ is compact. It is to say that a nilpotent cone $\sigma$ has a $\sigma$-nilpotent orbit if

$$
\exp (\sigma) \subset \overline{C(S)} \subset U(S)
$$

for some Satake boundary component $S$, and $\sigma$ has no $\sigma$-nilpotent orbit (i.e., $\left.D_{\sigma}=D\right)$ otherwise. Hence we have the following. 
TheOREM 5.6. Let $\sigma$ be a nilpotent cone in $\mathfrak{g}$. Then $E_{\sigma} \rightarrow \Gamma(\sigma)^{\mathrm{gp}} \backslash D_{\sigma}$ is trivial.

ExAmple 5.7. Let $D$ be the upper half-plane. $G=S L(2, \mathbb{R})$ acts on $D$ by linear fractional transformation. By the Cayley transformation, $D \cong \Delta:=$ $\{z \in \mathbb{C}|| z \mid<1\}$. Take the Satake boundary component $S=\{1\} \in \partial \Delta$. Then

$$
\begin{aligned}
& N(S)=\left\{\left(\begin{array}{cc}
u & v \\
0 & u^{-1}
\end{array}\right) \mid u \in \mathbb{R} \backslash\{0\}, v \in \mathbb{R}\right\}, \\
& W(S)=U(S)=\left\{\left(\begin{array}{ll}
1 & v \\
0 & 1
\end{array}\right) \mid v \in \mathbb{R}\right\}, \\
& C(S)=\left\{\left(\begin{array}{ll}
1 & v \\
0 & 1
\end{array}\right) \mid v \in \mathbb{R}_{\geq 0}\right\}
\end{aligned}
$$

(see $[\mathrm{N}])$. Take the nilpotent $N=\left(\begin{array}{ll}0 & 1 \\ 0 & 0\end{array}\right)$ and the nilpotent cone $\sigma=$ $\mathbb{R}_{\geq 0} N \subset \mathfrak{g}$. Here $\exp \left(\sigma_{\mathbb{C}}\right)=U(S)_{\mathbb{C}}$. The compact dual $\check{D}$ and the subspace $B(\sigma)=\exp \left(\sigma_{\mathbb{C}}\right) \cdot D \subset \check{D}$ are described as

$$
\check{D}=\mathbb{C} \sqcup\{\infty\} \cong \mathbb{P}^{1}, \quad B(\sigma)=\mathbb{C} .
$$

$\operatorname{Exp}\left(\sigma_{\mathbb{C}}\right) \backslash B_{\sigma}=\mathbb{B}(\sigma)$ is a point, and $B(\sigma) \rightarrow \mathbb{B}(\sigma)$ is a trivial principal bundle over $\mathbb{B}(\sigma)$. Take $F_{0} \in B(\sigma)$. We have a trivialization

$$
B(\sigma)=\exp \left(\sigma_{\mathbb{C}}\right) \cdot F_{0} \cong \exp \left(\sigma_{\mathbb{C}}\right) \times\left\{F_{0}\right\} .
$$

For $\Gamma=S L(2, \mathbb{Z})$

$$
\Gamma(\sigma)^{\mathrm{gp}}=\left\{\left(\begin{array}{ll}
1 & v \\
0 & 1
\end{array}\right) \mid v \in \mathbb{Z}\right\}=\exp (\mathbb{Z} N)
$$

is a lattice of $\exp \left(\sigma_{\mathbb{C}}\right)$, and $\mathbb{G}_{m} \cong \exp \left(\sigma_{\mathbb{C}}\right) / \Gamma(\sigma)^{\mathrm{gp}}$. Then we have the trivial principal $\mathbb{G}_{m}$-bundle $\Gamma(\sigma) \mathrm{gp} \backslash B(\sigma) \rightarrow \mathbb{B}(\sigma)$. By the torus embedding $\mathbb{G}_{m} \hookrightarrow$ $\mathbb{C}$, we have the trivial associated bundle

$$
\left(\Gamma(\sigma)^{\mathrm{gp}} \backslash B(\sigma)\right) \times_{\mathbb{G}_{m}} \mathbb{C} \cong \mathbb{C} \times\left\{F_{0}\right\}
$$

And we have

$$
E_{\sigma}=\left\{\begin{array}{l|l}
(q, F) \in \mathbb{C} \times B(\sigma) & \begin{array}{l}
\exp \left((2 \pi i)^{-1} \log (q) N\right) F \in D \\
F \in B(\sigma)
\end{array} \\
F \in 0 & \text { if } q=0
\end{array}\right\} .
$$


The map

$$
\begin{aligned}
& \left(\mathbb{C} \times\left\{F_{0}\right\}\right) \cap E_{\sigma} \hookrightarrow E_{\sigma} \rightarrow \Gamma(\sigma)^{\mathrm{gp}} \backslash D_{\sigma} ;
\end{aligned}
$$

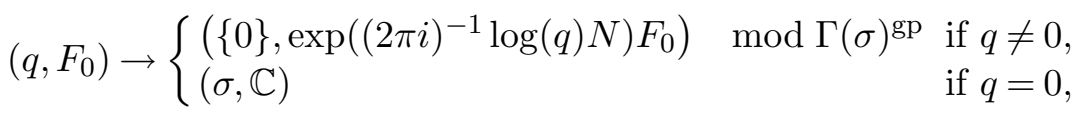

is an isomorphism by Lemma 5.3, and we have the following commutative diagram:

$$
\begin{gathered}
\left(\mathbb{C} \times\left\{F_{0}\right\}\right) \cap E_{\sigma} \cup E_{\sigma} \\
\cong \\
\Gamma(\sigma)^{\mathrm{gp}} \backslash D_{\sigma}
\end{gathered}
$$

Then the torsor $E_{\sigma} \rightarrow \Gamma(\sigma)^{\mathrm{gp}} \backslash D_{\sigma}$ has a section; that is, the torsor is trivial.

\subsection{The case where $E_{\sigma} \rightarrow \Gamma(\sigma)^{\mathrm{gp}} \backslash D_{\sigma}$ is nontrivial}

Let $w=3$, and let $h^{p, q}=1(p+q=3, p, q \geq 0)$. Let $H_{0}$ be a free module of rank 4 , and let $\langle,\rangle_{0}$ be a nondegenerate alternating bilinear form on $H_{0}$. In this case $D \cong S p(2, \mathbb{R}) /(U(1) \times U(1))$. Then $D$ is not a Hermitian symmetric space. Take $e_{1}, \ldots, e_{4}$ as a symplectic basis for $\left(H_{0},\langle,\rangle_{0}\right)$; that is,

$$
\left(\left\langle e_{i}, e_{j}\right\rangle_{0}\right)_{i, j}=\left(\begin{array}{cc}
0 & -I \\
I & 0
\end{array}\right)
$$

Define $N \in \mathfrak{g}$ as follows:

$$
N\left(e_{3}\right)=e_{1}, \quad N\left(e_{j}\right)=0(j \neq 3) .
$$

Proposition 5.8. Let $\sigma=\mathbb{R}_{\geq 0} N$. Then $E_{\sigma} \rightarrow \Gamma(\sigma)^{\mathrm{gp}} \backslash D_{\sigma}$ is nontrivial.

Proof. Define

$$
\left(u_{1}, \ldots, u_{4}\right):=\frac{1}{\sqrt{2}}\left(e_{1}, \ldots, e_{4}\right)\left(\begin{array}{cc}
I & I \\
i I & -i I
\end{array}\right)
$$

That is,

$$
\left(\left\langle u_{i}, u_{j}\right\rangle_{0}\right)_{i, j}=\left(\begin{array}{cc}
0 & i I \\
-i I & 0
\end{array}\right), \quad\left(\left\langle u_{i}, \overline{u_{j}}\right\rangle_{0}\right)_{i, j}=\left(\begin{array}{cc}
i I & 0 \\
0 & -i I
\end{array}\right)
$$


Take $F_{w}, F_{\infty} \in D(w \in \mathbb{C})$ as follows:

$$
\begin{aligned}
& F_{w}^{3}=\operatorname{span}_{\mathbb{C}}\left\{w u_{1}+u_{2}\right\}, \quad F_{w}^{2}=\operatorname{span}_{\mathbb{C}}\left\{w u_{1}+u_{2}, u_{3}-w u_{4}\right\}, \\
& F_{\infty}^{3}=\operatorname{span}_{\mathbb{C}}\left\{u_{1}\right\}, \quad F_{\infty}^{2}=\operatorname{span}_{\mathbb{C}}\left\{u_{1}, u_{4}\right\} .
\end{aligned}
$$

We have the maximally compact subgroup of $G$ at $F_{0}$

$$
\begin{aligned}
K & =\left\{g \in G \mid\left\langle C_{F_{0}}(g v), \overline{g w}\right\rangle_{0}=\left\langle C_{F_{0}}(v), \bar{w}\right\rangle_{0} \quad \text { for } v, w \in H_{\mathbb{C}}\right\} \\
& =\left\{\left(\begin{array}{cc}
X & 0 \\
0 & \bar{X}
\end{array}\right) \mid X \in U(2)\right\},
\end{aligned}
$$

where matrices in the second equation are expressed with respect to the basis $\left(u_{1}, \ldots, u_{4}\right)$. The $K$-orbit of $F_{0}$ is given by

$$
K \cdot F_{0}=K_{\mathbb{C}} \cdot F_{0}=\left\{F_{w} \mid w \in \mathbb{C}\right\} \sqcup F_{\infty} \cong \mathbb{P}^{1} .
$$

We assume that $E_{\sigma} \rightarrow \Gamma(\sigma)^{\mathrm{gp}} \backslash D_{\sigma}$ is trivial. Let $\varphi$ be a section of $E_{\sigma} \rightarrow$ $\Gamma(\sigma)^{\mathrm{gp}} \backslash D_{\sigma}$. We define a holomorphic morphism $\Phi: D \rightarrow \mathbb{C}$ such that

$$
\Phi: D \stackrel{\text { quot. }}{\longrightarrow} \Gamma(\sigma)^{\mathrm{gp}} \backslash D \hookrightarrow \Gamma(\sigma)^{\mathrm{gp}} \backslash D_{\sigma} \stackrel{\varphi}{\longrightarrow} E_{\sigma} \stackrel{\text { proj. }}{\longrightarrow} \text { toric }_{\sigma} \cong \mathbb{C} .
$$

Since $K F_{0} \cong \mathbb{P}^{1},\left.\Phi\right|_{K F_{0}}$ is constant.

On the other hand, $\left(\sigma, \exp \left(\sigma_{\mathbb{C}}\right) F_{0}\right)$ is a nilpotent orbit (it is easy to check the condition of Definition 3.1). Then

$$
\lim _{x \rightarrow \infty} \Phi\left(\exp (i x N) F_{0}\right)=0
$$

Define $N^{\prime} \in \mathfrak{g}$ as follows:

$$
N^{\prime}\left(u_{3}\right)=u_{1}, \quad N^{\prime}\left(u_{j}\right)=0(j \neq 3) .
$$

Then we have

$$
\begin{aligned}
& \exp (i x N) F_{0}=\exp \left(\frac{x}{2+x} N^{\prime}\right) F_{0} \\
& F_{\infty}=\exp \left(\frac{x}{2+x} N^{\prime}\right) F_{\infty}
\end{aligned}
$$

for $x \in \mathbb{R} \backslash\{-2\}$, and

$$
\exp \left(z N^{\prime}\right) K F_{0} \subset D \quad \text { for }|z|<1 \text {. }
$$


Then $\left.\Phi\right|_{\exp \left(z N^{\prime}\right) K F_{0}}$ is constant for each $|z|<1$, again because $\exp \left(z N^{\prime}\right) K \times$ $F_{0} \cong \mathbb{P}^{1}$. Finally we have

$$
\begin{aligned}
\Phi & \left(\exp (i x N) F_{0}\right) \\
& =\Phi\left(\exp \left(\frac{x}{2+x} N^{\prime}\right) F_{0}\right) \\
& =\Phi\left(\exp \left(\frac{x}{2+x} N^{\prime}\right) F_{\infty}\right) \\
& =\Phi\left(F_{\infty}\right)
\end{aligned}
$$

for $x>-1$. This contradicts condition (5.3), since $\Phi\left(F_{\infty}\right) \in \operatorname{torus}_{\sigma}$ if $F_{\infty} \in$ $D$.

Acknowledgment. Part of the main results here is based on the author's master's thesis. The author is grateful to Professors Sampei Usui and Christian Schnell for their valuable advice and warm encouragement. The author is also grateful to the referee for his careful reading and valuable suggestions and comments on the presentation.

\section{REFERENCES}

[AMRT] A. Ash, D. Mumford, M. Rapoport, and Y. S. Tai, Smooth Compactification of Locally Symmetric Varieties, Math. Sci. Press, Brookline, MA, 1975.

[CCK] J. A. Carlson, E. Cattani, and A. Kaplan, "Mixed Hodge structures and compactification of Siegel's space," in Journées de géométrie algébrique d'Angers, Juillet 1979 (Angers, France, 1979), Sijthohoff \& Nordhoff, Alphen aan den Rijn, 1980, 77-105.

[F] W. Fulton, Introduction to Toric Varieties, Ann. of Math. Stud. 131, Princeton University Press, Princeton, 1993.

[GGK] M. Green, P. Griffiths, and M. Kerr, "Néron models and boundary components for degenerations of Hodge structures of mirror quintic type," in Curves and Abelian Varieties, Contemp. Math. 465, Amer. Math. Soc., Providence, 2007, 71-145.

[K] K. Kato, "Logarithmic structures of Fontaine-Illusie," in Algebraic Analysis, Geometry, and Number Theory, Johns Hopkins University Press, Baltimore, 1989, 191-224.

[KU] K. Kato and S. Usui, Classifying Space of Degenerating Polarized Hodge Structures, Ann. of Math. Stud. 169, Princeton University Press, Princeton, 2009.

[N] Y. Namikawa, Toroidal Compactification of Siegel Spaces, Lecture Notes in Math. 812, Springer, Berlin, 1980.

[S] W. Schmid, Variation of Hodge structure: The singularities of the period mapping, Invent. Math. 22 (1973), 211-319.

[U] S. Usui, Generic Torelli theorem for quintic-mirror family, Proc. Japan Acad. Ser. A Math. Sci. 84 (2008), 143-146. 
Graduate School of Science

Osaka University

Osaka 560-0043

Japan

t-hayama@cr.math.sci.osaka-u.ac.jp 